\title{
Influences on the triple alpha process beyond the Hoyle state
}

\author{
Christian Aa. Diget ${ }^{*} \dagger$, Maria J. G. Borge ${ }^{b}$, Rafik Boutami $^{b}$, Peter Dendooven $^{c}$, \\ Tommi Eronen $^{d}$, Simon P. Fox ${ }^{e}$, Brian R. Fulton ${ }^{e}$, Hans O. U. Fynbo ${ }^{a}$, Henrik B. \\ Jeppesen $^{f}$, Ari Jokinen ${ }^{d}$, Björn Jonson ${ }^{g}$, Anu Kankainen ${ }^{d}$, lain Moore ${ }^{d}$, Arto \\ Nieminen $^{d}$, Solveig G. Pedersen ${ }^{a}$, Heikki Penttilä ${ }^{d}$, Victor F. E. Pucknell ${ }^{h}$, Karsten \\ Riisager $^{f}$, Sami Rinta-Antila ${ }^{d}$, Olof $\operatorname{Tengblad}^{b}$, Youbao Wang ${ }^{d}$, Katarina \\ Wilhelmsen $^{g}$, Juha Äystö ${ }^{d}$ \\ ${ }^{a}$ Institut for Fysik og Astronomi, Aarhus Universitet, DK-8000 Århus, Denmark \\ ${ }^{b}$ Instituto Estructura de la Materia, CSIC, E-28006 Madrid, Spain \\ ${ }^{c}$ KVI, Zernikelaan 25, 9747 AA Groningen, Netherlands \\ ${ }^{d}$ Department of Physics, University of Jyväskylä, FIN-40014 Jyväskylä, Finland \\ e Department of Physics, University of York, Heslington, YO10 5DD, UK \\ ${ }^{f}$ PH Division, CERN, CH-1211 Geneva 23, Switzerland \\ ${ }^{g}$ Fundamental Physics, Chalmers Univ. of Technology, S-41296 Göteborg, Sweden \\ ${ }^{h}$ CCLRC Daresbury Laboratory, Daresbury Warrington, Cheshire WA4 4AD, UK \\ E-mail: digetenscl.msu.edu
}

The triple alpha process is studied using indirect methods. The beta decays of ${ }^{12} \mathrm{~N}$ and ${ }^{12} \mathrm{~B}$ are used to probe the triple alpha continuum of ${ }^{12} \mathrm{C}$. Different independent breakup channels are identified, consistently showing that the $10 \mathrm{MeV}$ strength is dominated by a $0^{+}$state interfering with the Hoyle state ghost. The $13-14 \mathrm{MeV}$ region on the other hand is dominated by a $2^{+}$state.

International Symposium on Nuclear Astrophysics - Nuclei in the Cosmos - IX

25-30 June 2006

CERN

\footnotetext{
${ }^{*}$ Speaker.

†Present: National Superconducting Cyclotron Laboratory, Michigan State University, East Lansing, Michigan MI-48824, USA.
} 


\section{The triple alpha process at resonance}

The triple alpha process is the process where three alpha particles combine to form ${ }^{12} \mathrm{C}$. In the most important temperature range from $0.1 \mathrm{GK}$ to $2 \mathrm{GK}$ the reaction rate is dominated by the ${ }^{8} \mathrm{Be}$ ground state and of the Hoyle state [1] at $7.654 \mathrm{MeV}$ in ${ }^{12} \mathrm{C}$. At this temperature range both resonances are within the Gamow window, and since they are both narrow, the narrow resonance case applies when calculating the triple alpha reaction rate. Because of this, the analytical expression for the rate is very simple: it is proportional to the radiative width of the Hoyle state and the population of the state.

\section{The triple alpha reaction rate at extreme temperatures}

In the extreme temperature regions outside the $0.1-2$ GK range, the resonant reaction through the Hoyle state does not necessarily dominate the reaction rate. ${ }^{1}$ The important contributions in these regions are of course still natural parity states and the corresponding direct reactions. For the high temperature region the importance of the individual contributions is determined by the available states in ${ }^{12} \mathrm{C}$ and ${ }^{8} \mathrm{Be}$ within the Gamow window. The different possible contributions are $0^{+}$and $2^{+}$strength in ${ }^{8} \mathrm{Be}$ and $0^{+}, 1^{-}, 2^{+}$and $3^{-}$strength in ${ }^{12} \mathrm{C}$. These contributions are all listed and discussed by Buchmann and Barnes [2].

When taking states other than the ${ }^{8} \mathrm{Be}$ ground state and the Hoyle state into account, we need to know not only the resonance energy and the radiative width, but the total width as well. This is because the resonances are in general not narrow. It is crucial for the reaction rate at high temperatures which of the states are included and what we assume about these states. This is exactly the reason why the two commonly used rates NACRE [3] and Caughlan and Fowler [4] differ at the high temperatures. In the first a relatively narrow $2^{+}$resonance at low energy ( 9 $\mathrm{MeV}$ ) is included whereas in the other it is not. This state has been described theoretically by P. Descouvemont and D. Baye [5], following the ideas of Morinaga [6] in describing the Hoyle state as a prolate triple alpha cluster, and building a rotational band on this state. The $2^{+}$state in this energy region is seen as the first such rotational excitation. Such a state however remains undetected experimentally.

Here we will not discuss all possible contributions, but focus only on a few. Firstly we should be aware that because the states are very close to the threshold, we must take special care as threshold effects can be of importance. One such threshold effect is the appearance of ghosts [7]. A ghost of a narrow state arises when the width of the state is heavily energy dependent. So for example the shape of the ${ }^{8} \mathrm{Be}$ ground state is not a simple Breit-Wigner distribution. In the high energy tail of this distribution (around $1 \mathrm{MeV}$ above the state) the width of the state becomes very big, so at these energies the high energy tail of the ${ }^{8} \mathrm{Be}$ ground state is blown up. This is a general threshold effect and does also apply for the Hoyle state.

Having said this let us focus on the important possibility of a $2^{+}$state in ${ }^{12} \mathrm{C}$ just above the 7.275 MeV triple alpha threshold, and the properties of such a state. There have been two recent experimental identifications of such a low energy $2^{+}$continuum state: Bency John et al. [8] suggest

\footnotetext{
${ }^{1}$ Note that these temperatures are mainly relevant for scenarios where the triple alpha process does not set the temperature.
} 
a $2^{+}$contribution at $11.46(20) \mathrm{MeV}$ with a width of $0.43(10) \mathrm{MeV}$ probing the states by inelastic alpha scattering on ${ }^{12} \mathrm{C}$. Itoh et al. [9] in a similar experiment suggest a $1 \mathrm{MeV}$ wide $2^{+}$state around $10.5 \mathrm{MeV}$. The main difficulty in these experiments is that the selectivity of this approach is limited, and therefore a lot of other states are populated in the same energy region.

\section{Beta decay experiment at IGISOL}

As mentioned before alpha scattering experiments are limited by the population of many states in the region of interest around $10 \mathrm{MeV}$. In order to avoid this background during the last years we have performed a series of experiments at the ISOLDE-CERN (Switzerland) and IGISOL (Jyväskylä, Finland) facilities to probe the triple alpha continuum. Here beta decays of ${ }^{12} \mathrm{~N}$ and ${ }^{12} \mathrm{~B}$ have been used. These decays only populate $0^{+}, 1^{+}$and $2^{+}$states in ${ }^{12} \mathrm{C}$ due to the beta decay selection rules, which avoids most of the background from other resonances. Results of the first experiments have already been published, see [10-12].

We will here present a new set of data, taken at the IGISOL separator in Jyväskylä [13] where these two isotopes are produced in the Ion Guide Isotope Separator. The ${ }^{12} \mathrm{~B}$ or ${ }^{12} \mathrm{~N}$ nuclei were implanted in a thin foil. The foil was surrounded by three Double Sided Silicon Strip Detectors allowing us to detect all three alpha particles of the beta delayed triple alpha breakup. With these triple coincidences we are able to distinguish between and analyze data through the decay channels: ${ }^{8} \mathrm{Be}\left(0^{+} \mathrm{gs}\right) ;{ }^{8} \mathrm{Be}\left(0^{+}\right.$ghost $)$and ${ }^{8} \mathrm{Be}\left(2^{+}\right)$as shown in the following.

\section{Triple coincidence data}

A scatter plot of the complete set of triple coincidence data (16k events) taken during about 5 days of ${ }^{12} \mathrm{~N}$ beam time is shown in figure 1 . Vertically we have the sum energy of the three alpha

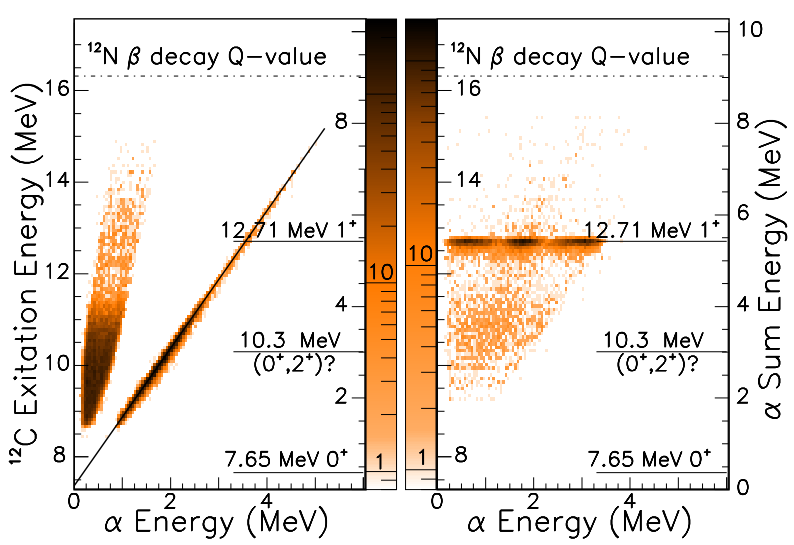

Figure 1: Triple coincidence data, beta delayed triple alpha breakup from ${ }^{12} \mathrm{~N}$. Two channels: Breakup through ${ }^{8} \mathrm{Be}$ ground state (left) and higher energies in ${ }^{8} \mathrm{Be}$.

particles, or equivalently the ${ }^{12} \mathrm{C}$ excitation energy. On the horizontal axis we have for any such event, the three individual alpha energies [14]. That is, one breakup event corresponds to three dots on a horizontal line. As can be seen to the right for the $12.7 \mathrm{MeV}$ state, this can be three very 
different energies. Or as seen to the left, one very high energy and two alpha particles sharing the remaining energy.

This shows directly some information on the breakup channel, since a narrow state-in this case the ${ }^{8} \mathrm{Be}$ ground state-determines the breakup directly by two body kinematics. The line superimposed on the data is exactly the line corresponding to the breakup through the ${ }^{8} \mathrm{Be}$ ground state just above the threshold, allowing us to look at these two breakup channels separately. In the right plot the situation is much more complicated, since it is not a clean sequential breakup. Furthermore this plot allows us to distinguish between the $0^{+}$or $2^{+}$states on the one hand and $1^{+}$ states on the other, since a $1^{+}$state cannot breakup through the ground state of ${ }^{8} \mathrm{Be}$ because of its unnatural parity, as is clearly seen for the $12.7 \mathrm{MeV} 1^{+}$state in ${ }^{12} \mathrm{C}$.

\section{The ${ }^{8}$ Be ground state channel}

Let us first look at the breakup through the ${ }^{8} \mathrm{Be}$ ground state: The spectra are fitted to an RMatrix model, as shown in figure 2. A three level model turns out to be essential to get a good fit.

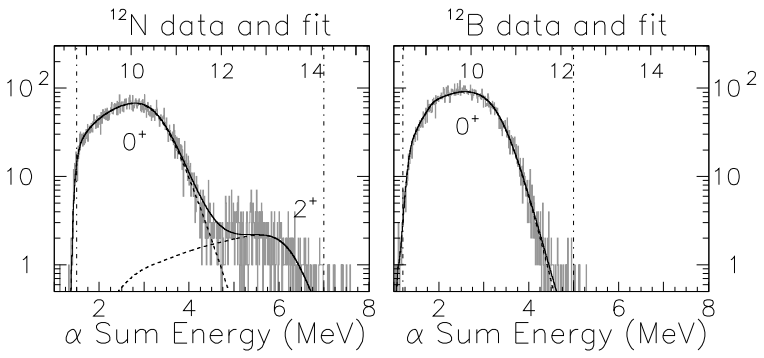

Figure 2: Measured spectra and R-Matrix fit for breakup through the ${ }^{8} \mathrm{Be}$ ground state, where $0^{+}$and $2^{+}$ contributions to the fits are shown. ${ }^{12} \mathrm{~N}$ and ${ }^{12} \mathrm{~B}$ beta delayed breakup.

The three states corresponding to these three levels are: A high energy $2^{+}$state at $13.61(14) \mathrm{MeV}$ of width 1.7(2) MeV dominating the upper energy region of the spectrum; a $0^{+}$state at an energy of $11.47(16) \mathrm{MeV}$ and of width 3.4(4) MeV; and the Hoyle state contributing with its ghost in this energy region, making interference between the two $0^{+}$states essential for understanding the spectrum. ${ }^{2}$ The two decay spectra are distorted by different beta decay phase space factors, and are influenced by the triple coincidence detection efficiency, however after correcting for these effects the two spectra are consistent and a combined fit of the two data sets is reasonable. Furthermore, the fit is consistent with our previously published data $[11,12]$ and is not consistent with a narrow $2^{+}$state at low energy.

\section{The ${ }^{8}$ Be excited state channel}

In case of a broad exit channel as for the higher energy region of ${ }^{8} \mathrm{Be}$, the situation is a bit more complicated. Here the efficiency dependens not only on the total energy of the three alpha particles, but on the way the three share the total energy. Since an analytical form of this detection efficiency is not available, it must be estimated using a Monte-Carlo simulation.

\footnotetext{
${ }^{2} \mathrm{As}$ in [12] the small contribution to the widths from the breakup through higher energies of ${ }^{8} \mathrm{Be}$ is neglected.
} 
The kinematics of the breakup are now determined not only by the energy in ${ }^{12} \mathrm{C}$ but as well by the line shape of the intermediate state of ${ }^{8} \mathrm{Be}$ and of the angular momentum in the breakups $\alpha+{ }^{8} \mathrm{Be}$ and $\alpha+\alpha$. In figure 3 the kinematics of the breakup at two different ${ }^{12} \mathrm{C}$ energies are

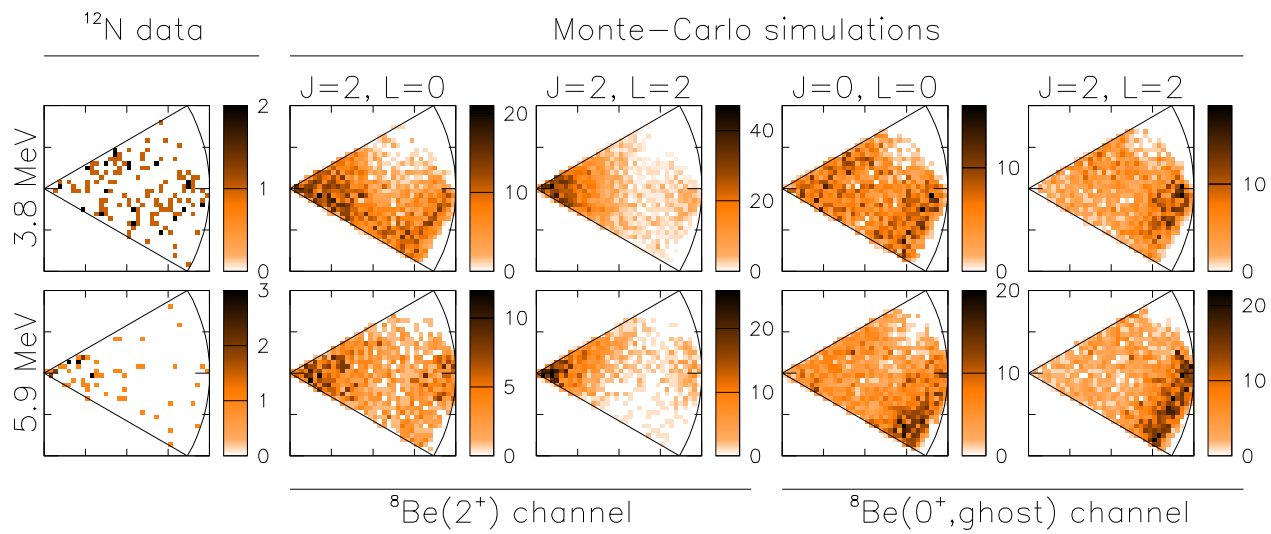

Figure 3: Dalitz plots for ${ }^{12} \mathrm{~N}$ data at two different sum energies. Simulated distributions for breakup through ${ }^{8} \mathrm{Be} 2^{+}$state and ${ }^{8} \mathrm{Be} 0^{+}$ghost. Two different possible spins $(J)$ of the involved ${ }^{12} \mathrm{C}$ state, two different angular momenta $(L)$ for the ${ }^{8} \mathrm{Be}\left(2^{+}\right)+\alpha$ intermediate system, where applicable. In addition to the simulations shown here, $J=0, L=2$ and $J=2, L=4$ breakups through the ${ }^{8} \mathrm{Be}\left(2^{+}\right)$state were as well simulated. These contributions however were easily excluded.

shown. The Dalitz plots [15] shown here are in fact only one sixth of a full Dalitz plot and ranges from 0 to 1 on the horizontal axis and from -0.5 to 0.5 on the vertical axis. These axis values are defined from the individual three alpha energies as: $\sqrt{3}\left(E_{1}-E_{3}\right) / E_{\text {sum }}$ and $\left(2 E_{2}-E_{1}-E_{3}\right) / E_{\text {sum }}$ respectively. The plots are shown for the ${ }^{12} \mathrm{~N}$ data as well as for Monte-Carlo simulations aimed at reproducing the observed distributions from a symmetrized R-Matrix description of the breakup [10].

The ${ }^{8} \mathrm{Be} 0^{+}$ghost channel is easily excluded for the high energy range as is the low energy $J=2, L=2$ breakup through the ${ }^{8} \mathrm{Be} 2^{+}$state. For the remaining a statistical analysis must be applied to discriminate between the different possibilities. With such an analysis, we can however conclude that at low energy the data is certainly dominated by breakup through the ${ }^{8} \mathrm{Be} 0^{+}$ghost, most likely from a $0^{+}$state in ${ }^{12} \mathrm{C}$. At the high energies on the other hand, a $2^{+}$state in ${ }^{12} \mathrm{C}$ is certainly dominating, and the breakup proceeds through the ${ }^{8} \mathrm{Be} 2^{+}$excited state, dominated by an orbital angular momentum of the first breakup of $L=2$. These two conclusions are consistent with those from the ${ }^{8} \mathrm{Be}$ ground state breakup channel.

\section{Summary and outlook}

Summarizing, we have used beta decay of ${ }^{12} \mathrm{~B}$ and ${ }^{12} \mathrm{~N}$ to probe triple alpha continuum states. With the used detector set-up, we have been able to detect triple alpha breakups, not only through the ${ }^{8} \mathrm{Be}$ ground state but through the ghost of this state as well as the excited $2^{+}$state of ${ }^{8} \mathrm{Be}$. Also, we have been able to distinguish between the channels, even the latter two. From this we have concluded that the $10 \mathrm{MeV}$ strength in ${ }^{12} \mathrm{C}$ is dominated by the interference between the $0^{+}$ 
Hoyle state and a wide $0^{+}$state just above $11 \mathrm{MeV}$ in ${ }^{12} \mathrm{C}$. Furthermore, the high energy region is certainly dominated by a $2^{+}$state in ${ }^{12} \mathrm{C}$.

Regarding the possibility of a $2^{+}$state, a narrow low energy $(9 \mathrm{MeV}) 2^{+}$state is not consistent with the measured data. A wider state $(1 \mathrm{MeV})$ at higher energies (e.g. $11 \mathrm{MeV})$ cannot be completely excluded from the data, but it must contribute little to the total spectrum. Such a $2^{+}$ state however would not dominate the triple alpha reaction rate at high temperatures, in contrast to a $2^{+}$state at a lower energy. We will therefore conclude that for the high temperature range the Caughlan and Fowler rate should be recommended. At low temperatures however, we would still recommend use of either the NACRE rate or the rate that we published recently [11].

It is worth noting that the data presented here were not sufficiently sensitive to the Hoyle state width to provide additional information on this. An intriguing outlook however is that if the ${ }^{12} \mathrm{C}$ spectrum (figure 2) had been measured with at least this precision at lower energies-if possible all the way down to the Hoyle state - the fitting of such a spectrum could prove to be sensitive to the Hoyle state width. This is because this width is not only determining the very narrow peak at 7.654 MeV but as well determines the ghost of the state and through the interference with the 11.5 $\mathrm{MeV} 0^{+}$state influences the ${ }^{12} \mathrm{C}$ spectrum. Such a spectrum has been measured recently and is presented in these proceedings by S. G. Pedersen [16].

\section{References}

[1] F. Hoyle et al., A State in C12 predicted from Astrophysical Evidence, in proceedings of The American Physical Society, Physical Review 92 (1953) 1095.

[2] L.R. Buchmann and C.A. Barnes, Nuclear reactions in stellar helium burning and later hydrostatic burning stages, Nuclear Physics A777 (2006) 254

[3] C. Angulo et al., A Compilation of Charged-Particle Induced Thermonuclear Reaction Rates, Nuclear Physics A656 (1999) 3.

[4] G.R. Caughlan and W.R. Fowler, Thermonuclear reaction rates, Atomic Data and Nuclear Data Tables 40 (1988) 283.

[5] P. Descouvemont and D. Baye, Microscopic theory of the $8 B e(\alpha, \gamma) 12 C$ reaction in a three-cluster model, Physical Review C36 (1987) 54.

[6] H. Morinaga, Interpretation of Some of the Excited States of 4n Self-Conjugate Nuclei, Physical Review 101 (1956) 254.

[7] F.C. Barker and P.B. Treacy, Nuclear levels near thresholds, Nuclear Physics, 38 (1962) 33.

[8] Bency John et al., Isoscalar electric multipole strength in 12C, Physical Review C68 (2003) 014305.

[9] M. Itoh et al., Study of the cluster state at Ex=10.3 MeV in 12C, Nuclear Physics A738 (2004) 268.

[10] H.O.U. Fynbo et al., Clarification of the Three-Body Decay of $12 \mathrm{C}(12.71 \mathrm{MeV})$, Physical Review Letters 91 (2003) 082502.

[11] H.O.U. Fynbo et al., Revised rates for the stellar triple-alpha process from measurement of $12 \mathrm{C}$ nuclear resonances, Nature 433 (2005) 136.

[12] C.Aa. Diget et al., Properties of the $12 \mathrm{C} 10 \mathrm{MeV}$ state determined through $\beta$-decay, Nuclear Physics A760 (2005) 3. 
[13] J. Ärje et al., The ion guide isotope separator on-line, IGISOL, Nuclear Instruments and Methods A247 (1986) 431

[14] H.O.U. Fynbo et al., The $\beta 2 p$ decay mechanism of 31Ar, Nuclear Physics $\mathbf{A 6 7 7}$ (2000) 38.

[15] R.H. Dalitz, On the analysis of $\tau$-meson data and the nature of the $\tau$-meson, The Philosophical Magazine 44 (1953) 1068.

[16] S.G. Pedersen, $\beta$-decay studies of states in 12C, in proceedings of International Symposium on Nuclear Astrophysics - Nuclei in the Cosmos - IX PoS(NIC-IX)244. 